\title{
REBOA as a Bridge to Brain CT in a Patient With Concomitant Brain Herniation and Haemorrhagic Shock - a Case Report
}

\section{Luca Bissoni ( $\sim$ luca.bissoni@gmail.com )}

Maurizio Bufalini Hospital: Ospedale Maurizio Bufalini https://orcid.org/0000-0002-6573-2312

\section{Emiliano Gamberini}

Maurizio Bufalini Hospital: Ospedale Maurizio Bufalini

\section{Lorenzo Viola}

Maurizio Bufalini Hospital: Ospedale Maurizio Bufalini

\section{Carlo Bergamini}

Maurizio Bufalini Hospital: Ospedale Maurizio Bufalini

\section{Emanuele Russo}

Maurizio Bufalini Hospital: Ospedale Maurizio Bufalini

\section{Giuliano Bolondi}

Maurizio Bufalini Hospital: Ospedale Maurizio Bufalini

\section{Vanni Agnoletti}

Maurizio Bufalini Hospital: Ospedale Maurizio Bufalini

\section{Case report}

Keywords: REBOA, TBI, Haemorrhagic shock, Brain hernation

Posted Date: May 20th, 2021

DOl: https://doi.org/10.21203/rs.3.rs-517648/v1

License: (c) (i) This work is licensed under a Creative Commons Attribution 4.0 International License. Read Full License

Version of Record: A version of this preprint was published at Trauma Case Reports on February 1st, 2022. See the published version at https://doi.org/10.1016/j.tcr.2022.100623. 


\title{
REBOA as a bridge to Brain CT in a patient with concomitant brain herniation and haemorrhagic shock - A case report
}

\author{
Luca Bissoni ${ }^{1}$, Emiliano Gamberini ${ }^{1}$, Lorenzo Viola ${ }^{1}$, Carlo Bergamini ${ }^{1}$, Emanuele Russo ${ }^{1}$, \\ Giuliano Bolondi ${ }^{1}$, Vanni Agnoletti ${ }^{1}$ \\ 1: Anesthesia and Intensive Care Unit, AUSL Romagna - Bufalini Hospital, Cesena (FC), Italy
}

Corresponding author:

Dr. Luca Bissoni, luca.bissoni@auslromagna.it phone +390547352818

\begin{abstract}
Introduction

The management of complex trauma patient with concomitant brain injury and extra-cranial lesions is challenging since the requirement of a low pressure to limit the bleeding clashes with the need to maintain an adequate cerebral perfusion and to obtain a brain CT-scan.

Here we present the use of REBOA as a bridge to CT scan in complex head and torso trauma.

Case presentation

A 59-years male patient involved in a car accident was admitted to our hospital after a car accident. He had a GCS of 3 with a left fixed pupil anisocoria. Despite right-sided chest decompression for pneumothorax and massive transfusion protocol for hemoperitoneum, blood pressure remained low; to temporally stabilize the patient and perform a brain CT scan a zone 1 REBOA was inserted and systolic blood pressure rose up from $60 \mathrm{mmHg}$ to $110 \mathrm{mmHg}$. A brain CT scan highlighted a right subdural hematoma with a 8-mm midline shift. The patient went to the operating room to perform damage control surgery and, subsequently, a decompressive craniotomy. After 96 days of hospital stay, the patient was discharged at home with a complete neurological recovery.

\section{Conclusions}

The achievement of a rapid brain CT scan in traumatic brain injury is often crucial and has a deep impact in changing surgical management; moreover, duration of cerebral herniation is associated with worse outcome and increased mortality.

In the light of this, the use of REBOA in selected cases of complex head and torso trauma could allow to gain time to go to the CT room in safe conditions.
\end{abstract}

\section{Background}

Uncontrolled haemorrhage is the principal cause of death among trauma patients ${ }^{1}$ and traumatic brain injury (TBI) is the leading cause of delayed mortality and disability ${ }^{2}$. Therefore the management of complex trauma with concomitant brain injury and extra-cranial lesions is a challenging situation associated with a high mortality rate.

In accordance with a recently published statement about the management of such a complex patient we have to face a major problem ${ }^{3}$ : damage-controlled resuscitation with permissive hypotension to reduce further haemorrhage and coagulopathy with the concomitant need of maintaining a cerebral perfusion pressure to prevent secondary brain damage with a chosen systolic blood pressure (SBP) of $100 \mathrm{mmHg}^{4}$. Moreover, in the management of a haemorrhagic trauma patient, the first thing to do is to stop the bleeding regardless of 
the presence of a non-haemorrhagic treat that could lead to severe disability and delayed mortality. In this kind of patient is often not possible (and it is not recommended) ${ }^{5}$ to obtain a Whole-Body CT-scan (WBCT) even if it has been shown that integration of a CT scan significantly increases the probability of survival of haemorrhagic unstable patients with blunt trauma ${ }^{6}$. The reasons are both clinical (reduce the time to treatment) and non-clinical (CT room's distance from the shock room) ${ }^{7}$.

Hybrid operative rooms, despite they are not present in every hospital, may represent a valuable solution to manage contemporary torso and head injuries after a rapid $\mathrm{WBCT}^{8}$.

Although damage control surgery (DCS) is the cornerstone of the treatment of haemorrhagic torso injury and is feasible without a CT scan, the management of a herniating brain with a decompressive craniotomy requires a brain $\mathrm{CT}$ scan.

Our hospital (HUB Bufalini Trauma Center, Cesena - AUSL Romagna, Italy) hosts a wellestablished trauma pathway composed by: a shock room where a primary survey is performed and life treating injuries are managed (airway management, chest decompression, resuscitative thoracotomy), a near-dedicated CT room that allows the execution of a fast WBCT with CT angiography and an emergency hybrid operative room where it is possible to perform DCS (operative and non-operative management).

We now present a case of a patient with concomitant TBI with incipient brain herniation and haemorrhagic shock in which we used a resuscitative endovascular balloon occlusion of the Aorta (REBOA) to stop the bleeding and perform a focused brain CT scan before going to the hybrid operative room for the DCS.

\section{Case presentation}

A 59-years male patient involved in a car accident was admitted to our shock room. The trachea was intubated on the field due to a GCS of 3 and the patient was mechanically ventilated. A left-side out-of-hospital chest decompression was performed. SpO2 at the shock room entering was $80 \%$ in $1 \mathrm{FiO} 2$. SBP was $60 \mathrm{mmHg}$. A left fixed pupil anisocoria was observed. The primary survey highlighted a right pneumothorax and a hemoperitoneum. A new right-sided chest decompression was performed with the restoration of normoxia. Hypotension remained unchanged despite massive transfusion protocol.

The trauma team decided to perform an emergent laparotomy and to temporarily stabilize the patient and perform an ongoing brain CT scan a zone 1 REBOA was inserted. After the positioning SBP rose up to 110 with a MAP of $80 \mathrm{mmHg}$. A rapid brain CT scan was performed and highlighted a right subdural hematoma with midline shift $>8 \mathrm{~mm}$. No others problems in patient management have been reported in the CT room.

Thereafter the patient went to the operating room. A DCS was performed with splenectomy and peritoneal packing. The total ischemia time was 30 minutes. Subsequently, a decompressive craniotomy was performed.

The overall patient's Injury Severity Score was 66. The total time spent in hospital was 96 days. By the time of the discharge, the patient had a complete neurological recovery.

\section{Conclusions}

We think that this case management could be a valid approach in the management of concomitant haemorrhagic shock and incipient cerebral herniation.

The optimum management is represented by a full hybrid shock room that allows the execution of both WBCT scan and surgical intervention. Kinoshita et al. reported that the use 
of a hybrid emergency room is associated with a decreased 28-days mortality in patients with severe trauma and reduced deaths by exsanguination ${ }^{9}$. Moreover, the same group found that the use of the hybrid emergency room is associated with the reduction of unfavorable outcomes in patients with severe $\mathrm{TBI}$, allowing a reduction in time required for diagnosis and intracranial surgery ${ }^{10}$.

In our context, the possibility of performing a brain CT in this selected kind of patients is often impossible and procrastinated to the end of the DSC for bleeding control.

The use of REBOA to manage haemorrhage is well described and is associated with improvement in the outcome of patients with torso injuries. Main complications described are ischemia, vessel ruptures, and vascular thrombosis ${ }^{11}$.

Its use in patients with concomitant TBI has been questioned because of the risk of a worsening of intracranial bleeding due to the increasing of the carotid flow and pressure. In an animal model of TBI and shock, REBOA increased the carotid flow and SBP but did not exacerbate TBI progression ${ }^{12}$. In the same study, massive transfusion was associated with the largest fluctuations of cerebral physiology variables, maybe related to important increases in CVP.

In our opinion REBOA placement is a safe procedure in a shocked patient where the treatment goals are to obtain a SBP improvement and a bleeding reduction, thus preserving cerebral perfusion pressure and reducing the impact of secondary brain damage ${ }^{13}$.

In conclusion, knowing that the duration of cerebral herniation is associated with worse outcomes and increased mortality ${ }^{8}$, the achievement of a rapid brain CT scan is fundamental and has a profound impact in changing surgical management, allowing concomitant brain decompression during DCS. In this selected case we obtained the brain CT with the use of a REBOA, which allowed us to gain time to go to the CT room in safe conditions.

Our approach is far from being the best one but is feasible, not time-consuming and quite safe, thinking at the kind of patients it is intended for. 


\section{LIST OF ABBREVIATIONS}

TBI: Traumatic Brain Injury

SBP: Systolic Blood Pressure

WBCT: Whole Body CT-scan

REBOA: Resuscitative Endovascular Baloon Occlusive

DCS: Damage Control Surgery

\section{KEYWORDS}

REBOA; TBI; Haemorrhagic shock; Brain hernation

\section{BIBLIOGRAPHY}

1 Ordoñez CA, Herrera-Escobar JP, Parra MW, et al. Computed tomography in hemodynamically unstable severely injured blunt and penetrating trauma patients. J Trauma Acute Care Surg. 2016;80(4):597-602; discussion 602-603.

2 Martino C, Russo E, Santonastaso DP, et al. Long-term outcomes in major trauma patients and correlations with the acute phase. World J Emerg Surg. 2020;15:6.

3 Picetti E, Rossi S, Abu-Zidan FM, et al. WSES consensus conference guidelines: monitoring and management of severe adult traumatic brain injury patients with polytrauma in the first 24 hours. World J Emerg Surg. 2019;14:53.

${ }^{4}$ Garvin R, Mangat HS. Emergency neurological life support: severe traumatic brain injury. Neurocrit Care. 2017;27(S1):159-169.

5 European Resuscitation Council (ERC): European Trauma Course http://www.erc.edu/courses/european-traumacourse. Accessed 26/02/2021

6 Huber-Wagner S, Mand C, Ruchholtz S, et al. Effect of the localisation of the CT scanner during trauma resuscitation on survival -- a retrospective, multicentre study. Injury. 2014;45 Suppl 3:S76-82.

7 Clarke JR, Trooskin SZ, Doshi PJ, Greenwald L, Mode CJ. Time to laparotomy for intra-abdominal bleeding from trauma does affect survival for delays up to 90 minutes. J Trauma. 2002;52(3):420-425.

8 Kinoshita T, Hayashi M, Yamakawa K, et al. Effect of the hybrid emergency room system on functional outcome in patients with severe traumatic brain injury. World Neurosurg. 2018;118:e792-e799.

9 Kinoshita T, Yamakawa K, Matsuda H, et al. The survival benefit of a novel trauma workflow that includes immediate whole-body computed tomography, surgery, and interventional radiology, all in one trauma resuscitation room: a retrospective historical control study. Ann Surg. 2019;269(2):370-376.

10 Otsuka H, Sato T, Sakurai K, et al. Effect of resuscitative endovascular balloon occlusion of the aorta in hemodynamically unstable patients with multiple severe torso trauma: a retrospective study. World J Emerg Surg. 2018;13(1):49.

11 Uchino H, Tamura N, Echigoya R, Ikegami T, Fukuoka T. "Reboa" - is it really safe? A case with massive intracranial hemorrhage possibly due to endovascular balloon occlusion of the aorta (Reboa). Am J Case Rep. 2016;17:810813.

12 Johnson MA, Williams TK, Ferencz S-AE, et al. The effect of resuscitative endovascular balloon occlusion of the aorta, partial aortic occlusion and aggressive blood transfusion on traumatic brain injury in a swine multiple injuries model. J Trauma Acute Care Surg. 2017;83(1):61-70. 
$13 \mathrm{Kim}$ DH, Chang YR, Yun J-H. Effects of resuscitative endovascular balloon occlusion of the aorta in neurotrauma: three cases. J Trauma Inj. 2020;33(3):175-180.

\section{DECLARATIONS}

\section{Acknowledgements}

Not applicable.

\section{Availability of data and materials}

Not applicable.

\section{Authors' contributions}

LB, EG and ER took care of patient in the Emergency Room and ICU and wrote and revised the paper. LV, CB, GB and VA revised the manuscript providing intellectual content.

\section{Funding}

This study did not receive any specific grants from any funding agencies in the public, commercial, and non-profit sectors.

\section{Ethics approval and consent to participate}

Not applicable.

\section{Consent for publication}

An informed consent for publishing the abovementioned data has been obtained in oral form by the patient.

\section{Competing interests}

The authors declare they have no competing interests. 